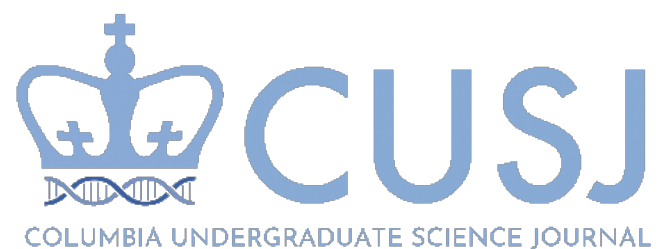

\title{
Microbiome Composition and Circadian Rhythm Disruption Alters Epithelial Barrier Integrity
}

\author{
Elisha Pinker ${ }^{1}$, Timur Tuganbaev² \\ ${ }^{1}$ Columbia University, New York, NY, USA \\ ${ }^{2}$ Lab of Dr. Eran Elinav; Department of Immunology, Weizmann Institute of Science, Israel \\ KEYWORDS: Circadian rhythms, intestinal permeability, microbiome
}

ABSTRACT: The intestine is home to one of the most complex ecological communities, termed the human gut microbiome. The gut microbiome modulates a wide range of human diseases from diabetes to neurological disorders to cancer. Separating the host and the gut microbiome is the epithelial barrier. The intestinal epithelium serves as an adaptive interaction hub between the host and microbiome that plays an important role in deciding the outcome of host-microbiome interactions. Regulation of epithelial barrier permeability to ions, nutrients and microbiome metabolites is known to be a tightly controlled process on the host side. While previous studies have identified the microbiome as a factor for epithelial permeability, the exact mechanisms through which it mediates remains less clear. Here, we show that alterations in microbiota composition by treatment with antibiotics selectively targeting specific members of the microbiome community impacts the permeability of the intestine. Additionally, modulating the microbiome through other methods such as altering diet composition shows changes in permeability of the epithelial barrier. As daily feeding rhythm entrains diurnal fluctuations in microbiome, we have set out to measure how epithelial barrier permeability disrupts the clock. We have discovered that the permeability of the intestinal epithelial barrier exhibits circadian rhythms in mice. Disruption of these rhythms, through jet lag or genetic deficiencies in circadian machinery, alters epithelial barrier integrity. Together, these findings provide evidence that disruptions in circadian rhythms as well as alterations in microbiome composition have direct consequences in intestinal permeability, and that microbiome might serve as a tool in regulating epithelium permeability.

\section{INTRODUCTION}

The human body is colonized by a complex community of microorganisms, termed the human microbiome, that includes bacteria, archaea, viruses and fungi [1]. This ecological community exists primarily on mucosal sites, like the gastrointestinal tract, skin, lungs and urogenital system [2]. This community is such where the microbiome bacteria outnumber eu- karyotic cells in the entire human body 1.3:1 [3]. The microbiota is responsible for the regulation of many physiological processes, including digestion, absorption [4], host metabolism [5], the maturation and function of the immune system [4], and even host behavior and cognitive function [6]. However, when the balanced microbial composition is disrupted, dysbiosis can cause intestinal diseases [7]. Modulating

(C) 2021 Pinker, Tuganbaev. This is an open access article distributed under the terms of the Creative Commons Attribution License, which permits the user to copy, distribute, and transmit the work provided that the original authors and source are credited. 
the microbiome through the diet is one way to induce dysbiosis. Treatment with high-fat diet (HFD), containing $60 \%$ of caloric energy derived from fat (a model of a "western diet"), is one such example where the balanced gut microbial community is altered to the point of dysbiosis resulting in metabolic dysfunction consequently inducing disease, such as obesity and type 2 diabetes as well as exacerbating inflammatory bowel diseases (IBD) [7]. But, when treated with broad-spectrum antibiotics, obesity and glucose intolerance in mice fed with HFD is abrogated [8]. Previous studies show, that when microbiome from mice fed HFD were transplanted into germ-free mice (GF: free of microorganisms) significantly more weight is put on by these mice compared to GF mice transplanted with microbiome from lean donor mice fed NC (normal chow) [9]. Additionally, GF condition in mice causes significant immune system deficiency [7]. Tools like gnotobiotic animal models (GF mice) and broad-spectrum antibiotics, have enabled the discovery that microbiota contributions to health and disease span multiple organ systems.

It has previously been shown that the mammalian intestinal microbiota composition exhibits diurnal oscillations both in terms of its composition and metabolic activity [8]. The circadian clock is the top of a hierarchical internal clock system that is responsible for the circadian biological processes ranging from dictating the rhythmic gene expression in organs to behavior, metabolism and immunity [10][11]. When working with circadian rhythms, scientists devised Zeitgeber times (ZT) which measure time on a 24-hour light/dark cycle, so ZT12 and ZT0 denote "dusk" and dawn", respectively. The molecular mechanisms behind the circadian clocks in mammals is orchestrated under a transcriptional autoregulatory feedback loop comprised of the 'core' clock genes CLOCK and BMAL1, responsible for encoding activators, and PER1, PER2, CRY1 and CRY2, responsible for encoding repressors [12]. A disruption of the circadian clock in hu- mans, environmentally or by gene deficiency, is associated with an array of diseases such as, obesity and diabetes [13] [14], cancer [15], cardiovascular disease [16], IBD (inflammatory bowel disease) exacerbation [17] and susceptibility to infection. One common feature among the diseases associated with circadian rhythm disruption, is that they appear to be triggered or promoted by inflammatory processes. Desynchronized interaction between mucosal organs and a complex community of microorganisms colonizing them have emerged as a potential source of inflammation underlining diseases promoted by circadian clock disruption. The source of this inflammation can be located at the gut epithelium [18]. The intestinal epithelium is comprised of a single-cell layer constituting the largest and most important barrier against the external environment [19]. Acting as a selectively permeable barrier, the intestinal epithelium can permit the absorption of nutrients, electrolytes and water, while simultaneously defending against toxins [19]. The epithelium maintains an effective barrier through the help of protein-protein networks forming complexes like tight junctions [19]. A compromised epithelial barrier allowing the passage of toxins, antigens and bacteria into the blood stream creates what is commonly known as "leaky gut" [20]. There is therefore an urgent need to better identify the players behind the disruption and upkeep of intestinal barrier function, to devise methods to combat negative barrier alteration. In this project we studied different factors affecting permeability in the epithelial barrier, specifically in the small intestine, the part of the intestine responsible for the majority of nutrient absorption [21]. Recent studies on type 2 diabetes and obesity have elucidated the link between microbiome composition and small intestine permeability; however, the mediators of these effects have yet to be determined [22]. Over the course of this project, we demonstrated the consequences that genetic and environmental circadian disruption have on gut barrier integrity in mice. Furthermore, we 
found evidence to suggest that altering the microbiome, either with diet intervention or antibiotic treatment, can disrupt the diurnal oscillations and alter permeability. Understanding the key players behind circadian disruption and intestinal barrier maintenance has potential in therapeutic and preventative medicine interventions.

\section{METHODS}

\section{Mice}

Under normal conditions, mice had strict lightdark cycles lasting 12 hours each. Lights were turned on either at 11:30 AM or 11:30 PM, ZT0 or ZT12 respectively. For the induction of jet lag, mice were shifted between both control light conditions every 3 days for 3 weeks at a consistent but arbitrary time. Jet lag mice were moved between the different light schedules every 3 days for 3 weeks. For consistency, jet lag mice were only experimented on at 1:30 PM, which was synchronized with the ZTs of the control group (i.e., ZT14 of jet lag mice corresponded to ZT14 of control mice, as all mice were exposed to the same light-dark conditions at the onset of sample collection). HFD mice were placed in ZT2 and ZT14 light-dark cycles to measure two time points upon sacrifice. These mice were fed a strict HFD for 3 weeks. Mice receiving antibiotic treatment, either received exclusively vancomycin (1 g/l), ampicillin (1 g/l), neomycin (1 $\mathrm{g} / \mathrm{l})$, and metronidazole $(1 \mathrm{~g} / \mathrm{l})$ or a combination of all four in drinking water for 3 weeks. Per 1/2/- (KO) and Per1/2-/+ (WT) were housed under normal conditions and sacrificed at ZT 14.

\section{Fecal Microbiome Transplantation (FMT)}

Microbiome samples were collected and stored in Eppendorf tubes containing 20\% glycerol. Fecal samples were homogenized and stored in dry ice before long-term storage at $-80^{\circ} \mathrm{C}$. For the microbiome transplantation, $0.1 \mathrm{~g}$ of thawed fecal sample was mixed in $1 \mathrm{~mL}$ sterile PBS and $200 \mu \mathrm{L}$ of the diluted sample was gavaged per mouse. HFD microbiome samples were taken from mice fed ad libitum HFD for 4 weeks.
Measuring Permeability with FITC-dextran

Food was removed from mice 2 hours before gavage (starting at $9 \mathrm{AM}$ ). The solution was made with $4 \mathrm{kDA}$ fluorescein isothiocyanate (FITC)-dextran dissolved in a phosphate buffered saline (PBS) with a concentration of $40 \mathrm{mg} / \mathrm{ml}$. At $11 \mathrm{AM}$, mice were gavaged with $200 \mu \mathrm{l}$ dextran. After 2.5 hours (1:30 PM, ZT14), blood was drawn and centrifuged to recover the serum. The fluorescence of the serum was quantified at an excitation wavelength of $485 \mathrm{~nm}$ and emission wavelength of $533 \mathrm{~nm}$ using a microplate reader.

\section{Ussing Chamber}

The Ussing chamber is an instrument used to measure epithelial resistance which is a measure of epithelial barrier permeability. The procedure followed was in accordance with the manufacturer's instructions (Warner Instruments, P2300). Small intestine tissue is excised and immediately mounted in the chamber. Current is then applied to the system and voltage clamp recordings were taken. Small intestine was carefully cleaned and handled minimally to prevent microfracturing the tissue and compromising the results. Additionally, samples were mounted within three hours of tissue excise to limit cell death.

\section{PRR stimulation measures degree of mi- crobial presence at systemic sites}

PRR reporter cell lines were obtained from Invivogen (HEK-Blue TLR (Toll-like Receptors) and NLR (Nod-like Receptors) reported cell lines): TLR2, TLR3, TLR4, TLR5, TLR7, TLR9, NOD1, NOD2. Extracts from spleen, liver, and serum were homogenized and added to reporter cell lines incubated with KEL-Blue detection medium according to manufacturer's instructions. Detection is a measurement of specific binding indicating extent of microbial leakage out of the gut.

\section{Statistical Analysis}

Data is presented as the mean \pm standard deviation. Mann-Whitney U-tests were per- 
formed to compare groups. ANOVA with post hoc test was used for comparison between multiple groups with a Bonferroni correction. $p<0.05$ and $q<0.1$ were the threshold for being considered significant. ${ }^{*} p<$ $0.05,{ }^{* *} p<0.01,{ }^{* * *} p<0.001,{ }^{* * * *} p<0.0001$.

\section{RESULTS}

\section{State of Small Intestinal Microbiome Deter- mines Epithelial Barrier Function}

With a GF mouse model, we initially set out to establish the role a microbiome has on the permeability of the small intestine epithelial barrier. Mice underwent fecal transplantation of either normal chow (NC) microbiome or high fat diet (HFD) fed donor specific pathogen free (SPF) microbiome, while the control group received phosphate buffered saline (PBS) (Figure 1A). Small intestine samples were taken and epithelial resistance was measured with the Ussing Chamber. Interestingly, mice with HFD microbiome showed increased permeability while mice with PBS (no microbiome) showed reduced permeability (Figure 1B). Together, this data suggests that microbiome shaped by diet is an active influencer over intestinal permeability.

We next set out to further analyze which subsets of bacteria within the microbiome community are most influential in barrier function modulation. With an antibiotic murine model, we could target specific subsets of bacteria to determine the impact the presence or lack thereof has on epithelial permeability in the intestine. We treated 5 groups of WT mice, fed with normal chow, with selective antibiotics that target specific members of microbiome: metronidazole (anaerobic bacteria), vancomycin (Gram-positive bacteria), ampicillin (broad spectrum), neomycin (Gram-negative bacteria), or a combination of all four for a duration of 3 weeks delivered in their drinking water (Figure 1C). The group containing all four antibiotics is a crude simulation of the germ-free mouse model. After 3 weeks, a FITC-dextran assay was performed, which entails orally ad- ministering mice with fluorescent dye coated $4 \mathrm{kDa}$ beads and measuring fluorescence in the peripheral blood, at ZT14, as a proxy for epithelial barrier permeability. Significant reduction in optical density (OD) was observed in four of the antibiotic groups compared to the control (Figure 1D). Additionally, vancomycin treated mice had significantly higher OD than neomycin and metronidazole groups (Figure 1D). The lack of a significant reduction in permeability in the combination treatment, containing all four antibiotics, was likely due to outliers. Two days after blood was taken, and fluorescence was no longer detectable, mice were sacrificed, at ZT14, and small intestine samples were removed for the Ussing Chamber measurements. Only the jejunum region of the small intestine was used for Ussing Chamber. All five antibiotic groups showed significantly reduced permeability and vancomycin, again, showed higher permeability than two of the other antibiotic groups (Figure 1E).

Together, these results uncover that the state of microbial composition directly alters the barrier integrity in the small intestine and the entire system. Additionally, vancomycin-treated groups experienced the smallest reduction in permeability suggesting, that gram-positive bacteria play the smallest role in intestinal epithelial barrier function compared to other bacterial subtypes in the gut microbiome.

\section{Genetic and Environmental Disruption of Circadian Clocks Impacts Epithelial Barrier Integrity}

It has been shown that a functional circadian clock of a host is required for normal diurnal rhythmicity in microbiota composition and function [8]. Building off our previous observations, the next step is to examine if circadian clock disruption will likewise compromise the intestinal epithelial barrier. Testing of Per1/2-/- mice, genetically deficient in a functional host clock, has previously shown to disrupt diurnal rhythmicity in microbiota composition [8]. With our own group of Per1/2-/- mice, we ran a FITC-dextran assay. 
A

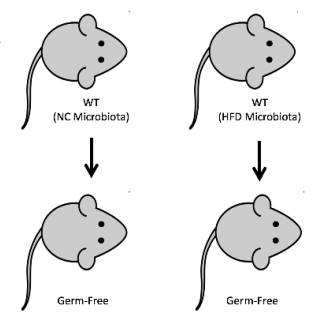

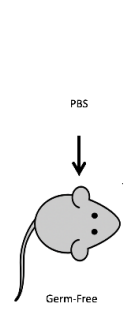

C

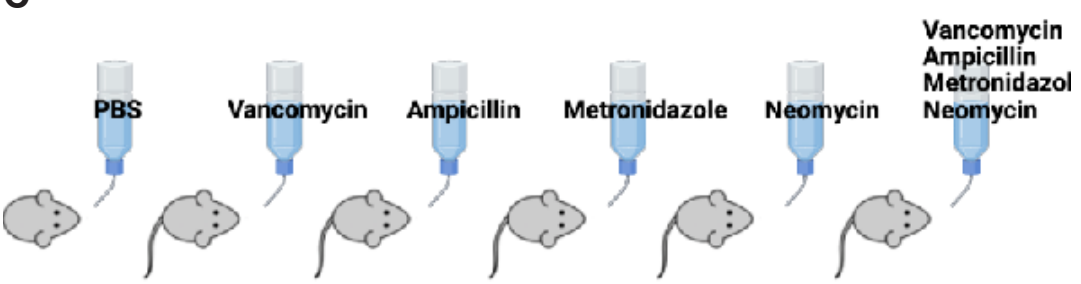

B

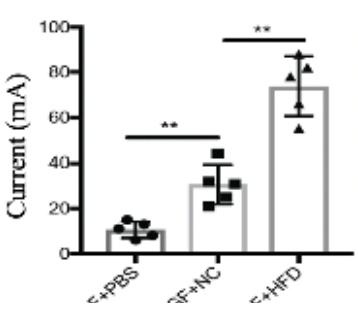

D
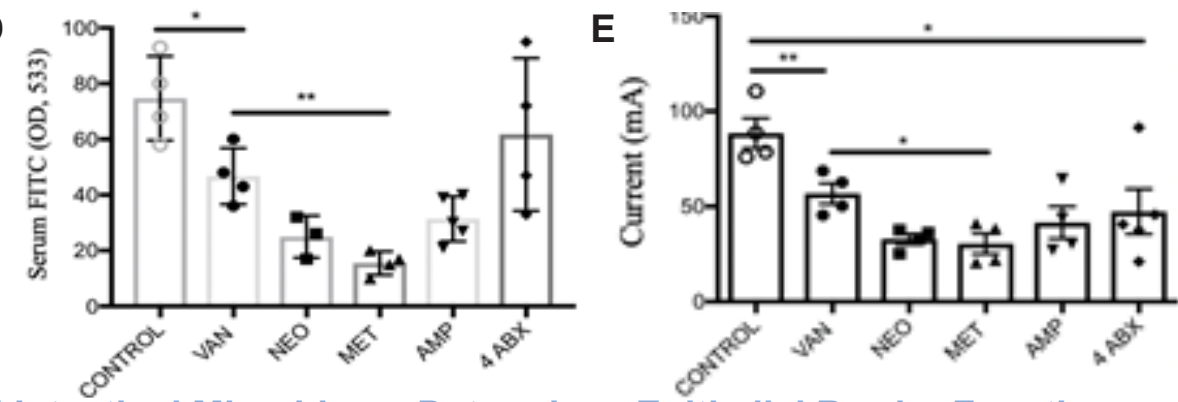

Figure 1. State of Small Intestinal Microbiome Determines Epithelial Barrier Function.

(A) Schematic summarizing transplantation of microbiota from HFD, NC and PBS into germ-free recipient mice.

(B) Ussing Chamber recording of small intestines from GF mice; measuring current ( $\mathrm{mA}$ ) as a measurement of permeability.

(C) Schematic showing a murine antibiotic treatment setup.

(D) FITC (fluorescein isothiocyanate)-dextran recovered from the serum of antibiotic treated WT mice and control.

(E) Ussing Chamber recording of small intestines from antibiotic treated WT mice and control.

Means \pm SD are plotted. ${ }^{*} p<0.05,{ }^{* *} p<0.01,{ }^{* * *} p<0.001,{ }^{* * * *} p<0.0001$ by Mann-Whitney $U$ test.

The OD for Per 1/2-/- was significantly higher (Figure 2A) demonstrating that circadian clock disruption results in increased intestinal permeability, a feature of a "leaky gut" syndrome.

Environmental clock disruptions such as chronic jet lag and shift work are behavioral patterns associated with an increased risk for obesity, diabetes and cardiovascular disease [23][18][14][16][24]. It has been previously established that a loss of microbiota oscillations and dysbiosis is associated with jet lag in mice [8]. So, we set out to learn if these losses of oscillations and dysbioses, in jet lag mice, also impact barrier function in the intestinal epithelial barrier. Prior to our jet lag experiment, we performed a 24-hour circadian experiment on WT mice measuring intestinal permeability with two independent methods - one measuring the electrical permeability of the epithelium to ions (the Ussing Chamber assay) and one measuring the concentration of microbial products in peripheral blood (Toll-like Receptor (TLR) assay) (Figure 2B, Figure 2C). Our findings demonstrate that just like microbial composition behaves in diurnal oscillations, so too does permeability. From this data we chose ZT2 and ZT14 as the lowest and highest points of permeability respectively and prepared control groups at each of these time points. This makes sense due to the nocturnal behaviors of mice where ZT2 is during the daylight when mice are least active while ZT14 is during the night is when mice are most active.

We then set out to mimic the situation of shift work and chronic jet lag by using a jet lag model in which mice were exposed to a 12-hour time shift every 3 days. Using a circadian cabinet, which allows us to individually control the light cycles of each compartment, a group of mice were initially started in one of two 
compartments (ZT2 or ZT14) with light cycle's 12-hours apart. Every 3 days for 3 weeks the cages of mice were switched between the two compartments until they were "jet lagged". The mice were then bled for FITC-dextran assay at the same time, ZT2 and ZT14, depending on the compartment. However, if the mice were jet lagged properly, and their circadian clock was disrupted, the ZT time at which blood was drawn would not matter. The results showed that the OD from FITC-dextran between jet lag groups ZT2 and ZT14 were non-significant (Figure 2D). Additionally, compared to the controls, the jet lag groups' ODs was closer to Control ZT14 (highest measured permeability) than Control ZT2 (lowest measured permeability). When we excised small intestine samples, for Ussing chamber assay, we observed similar trends: non-significant permeability measurements between jet lag groups (sign of successful jet lag) and overall permeability levels aligned with ZT14 control levels (Figure 2E).

Altogether, these data suggest that chronic environmental (jet lag model) or genetic disruption (Per1/2-/-) of host light-dark cycles exhibits significant alterations in intestinal epithelial barrier function and as a result increasing barrier permeability. This has relevance for frequent fliers traveling across different time zones and people who perform shift work. Additionally, our data suggests an explanation for clinical observations, finding shift workers, with irregular sleep/wake schedules, to experience exacerbated inflammatory bowel disease (IBD) due to the effect endogenous misalignment of circadian rhythms has on intestinal homeostasis [26].

\section{Metabolic Syndrome Induced by HFD Re- sults in Circadian Disruption and Intestinal Barrier Dysfunction}

Metabolic syndromes, like obesity and diabetes, have impacted hundreds of millions of people worldwide and consequently taken the lives of millions annually [25]. Previous studies have uncovered the associations metabolic syndromes have with the functionality of the intes-
A

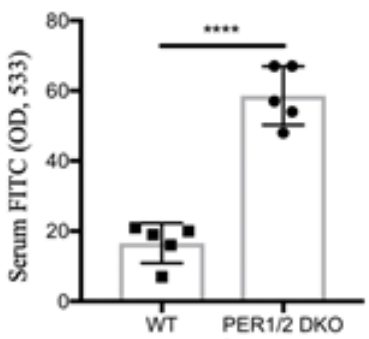

B

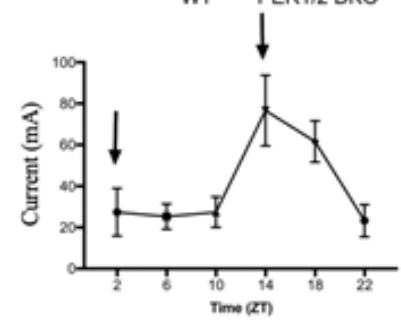

C

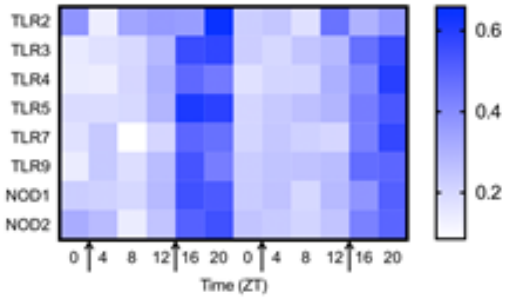

E
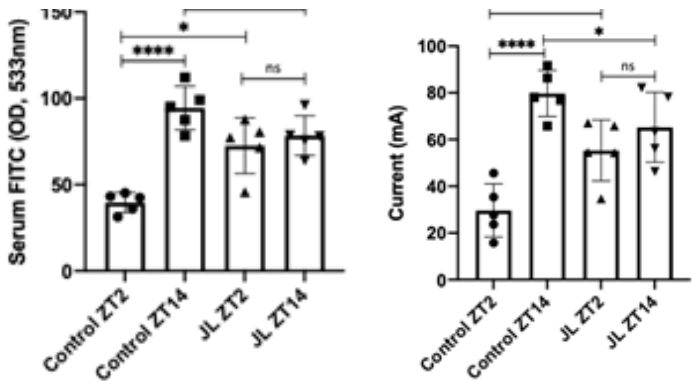

Figure 2. Genetic and Environmental Disruption of Circadian Clocks Impacts Epithelial Barrier Integrity.

(A) FITC (fluorescein isothiocyanate)-dextran recovered from the serum of Per1/2-/- and WT.

(B) Ussing Chamber recording of small intestines from naïve WT over 24 hours. Arrows mark the lowest (ZT2) and highest (ZT14) points of permeability.

(C) PRR stimulation by spleen, liver, serum extracts from naïve WT mice. Arrows mark the lowest (ZT2) and highest (ZT14) points of permeability.

(D) FITC (fluorescein isothiocyanate)-dextran recovered from the serum of jet lag mice and their respective controls.

(E) Ussing Chamber recording of small intestines from jet lag mice and their respective controls. Means \pm SD are plotted. ${ }^{*} p<0.05,{ }^{* *} p<0.01,{ }^{* * *} p$ $<0.001,{ }^{* * * *} p<0.0001$ by Mann-Whitney $U$ test. Figures 2D and 2E: 2-way ANOVA test. 
tinal barrier, specifically its state of permeability [27]. The study highlights the implications an influx of immune-stimulatory microbial ligands, leaking out of a permeable intestinal barrier can have, such as increased inflammation and risk of infection [27]. However, the tissue under scrutiny was the colon. In our studies, we focused our attention on the small intestine, which one could argue is of more significance due to it being the primary site of nutrient absorption [21]. In order to mimic human dietary habits, which would predispose one to a metabolic syndrome, we fed mice with an HFD for 3 weeks. In a previously referenced chart (Figure 1B), where germ-free mice received fecal transplantation of an HFD microbiome we saw a significant increase in small intestine permeability than mice with a normal diet (NC) microbiome, suggesting that a microbial composition associated with an HFD diet causes intestinal barrier dysfunction.

Previous studies linked circadian rhythm disruption to metabolic syndrome when they found enhanced weight gain and exacerbated glucose intolerance in jet lag mice fed
HFD [8]. In an attempt to determine if an HFD would alter permeability in a circadian context we set out to perform another permeability experiment on HFD mice but under circadian conditions. We fed two groups of mice with HFD for 3 weeks. Utilizing the circadian cabinet, each group was placed in different compartments and light-dark cycles were set 12-hours apart at the previously observed lowest and highest times of permeability, ZT2 and ZT14 respectively (Figure 3A). FITC-dextran assay was run and blood was drawn at ZT2 and ZT14 depending on which compartment the mice were in. From the FITC-dextran data we see no significant difference in OD between HFD diet groups which suggests that the altered diet disrupted circadian rhythms (Figure 3B). Additionally, we see OD levels of HFD groups to be at levels closer to Control group ZT14 reaffirming previous data showing increased permeability in HFD mice (Figure 3B). When the Ussing Chamber is performed on small intestine samples, we likewise see no significance between HFD groups and overall HFD permeability levels correlate
A

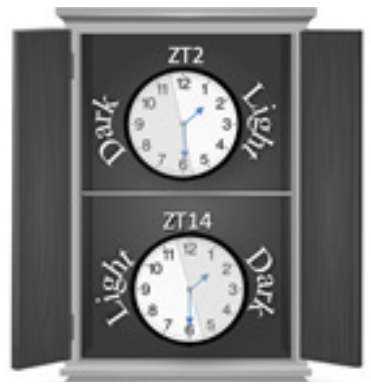

B

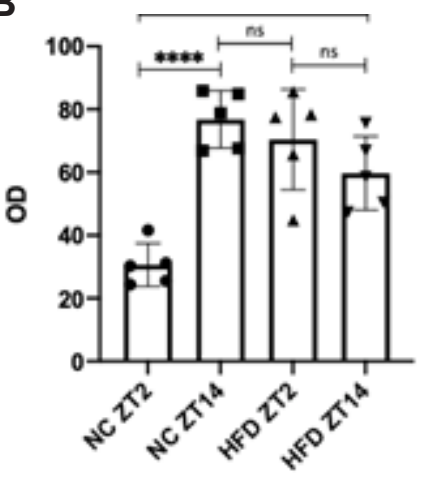

C

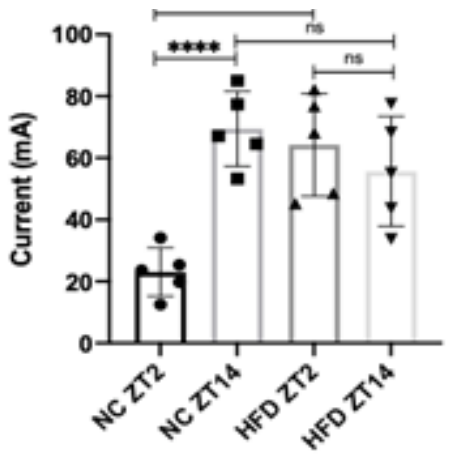

Figure 3. Metabolic Syndrome Induced by HFD Results in Circadian Disruption and Intestinal Barrier Dysfunction.

(A) Schematic showing the typical configuration of a circa-dian cabinet.

(B) FITC (fluorescein isothiocyanate)-dextran recovered from the serum of HFD mice at different ZT times and their respective controls.

(C) Ussing Chamber recording of small intestines of HFD mice at different ZT times and their respective controls.

Means \pm SD are plotted. ${ }^{*} p<0.05,{ }^{* *} p<0.01,{ }^{* * *} p<0.001,{ }^{* * *} p<0.0001$ by Mann-Whitney $U$ test. Figure 3B and 3C: 2-way ANOVA test. 
more strongly with ZT14 than ZT2 (Figure 3C). Together, these observed results draw attention to a serious issue. Metabolic syndromes, which are usually onset by poor diets such as those high in fat, are only exacerbated by the same stimuli that caused the development of the disease. Our data signifies metabolic syndromes' role in both disrupting circadian rhythms and heightening intestinal barrier permeability that can lead to increased inflammation and infection.

\section{DISCUSSION \& CONCLUSION}

In this study, we describe that the mammalian intestinal barrier function displays diurnal oscillations and is dependent on microbiome composition. If microbiome composition is distorted, as in the case of antibiotic treatment or HFD, we see alterations in intestinal barrier integrity as well as disruptions in the circadian rhythms of epithelial permeability. While previous studies sought to understand the consequences, diurnal disruption has on microbial composition, here we found direct evidence of the role microbial composition plays in intestinal permeability. Next, we wanted to study the direct implications distorting circadian rhythms, either with genetic clock deficiency or time-shifted induced jet lag, have on intestinal barrier function. We not only found that these conditions significantly impaired the circadian rhythms of permeability, but also, increased overall permeability resulting in greater pathobiont exposure and inflammation. Lastly, we found that inducing metabolic syndromes via HFD intervention disrupts circadian rhythms in the intestinal barrier as well as causes significant barrier dysfunction resulting in increased epithelial permeability.

Altogether, these studies have uncovered numerous future paths. While this study has broadly implicated microbiome composition as a mediator of gut permeability, in order to learn more about the key bacterial players in permeability mediation, we can perform sequencing on the microbial compositions found in our antibiotic treated groups to reveal the unique bacteria having the biggest impact on reducing or increasing permeability. Understanding which bacteria to promote and which to remove in the microbiota could be pivotal in suppressing symptoms in patients with IBD and metabolic syndromes, to name a few, caused by leakages in the intestinal epithelial. Exploiting antibiotic or probiotic treatments to target these bacteria could help combat diseases exacerbated by gut leakiness. Lastly, our observations showing intestinal permeability functioning under diurnal oscillations has applications from drug consumption to nutritional/dietary strategies. Further unravelling the roles, regulators and mechanisms of intestinal permeability could serve to one day aid therapies for illnesses affected by the "leaky gut" syndrome.

\section{AUTHOR INFORMATION}

\section{Corresponding Author}

*Elisha Pinker

ep2858@columbia.edu

\section{Funding Sources}

Columbia College Class of 1939 Fellowship

\section{Competing Interests}

The authors declare no competing financial and non-financial interests.

\section{ACKNOWLEDGMENTS}

E.P. would like to thank Columbia College and the Weizmann Institute of Science for supporting this research.

\section{REFERENCES}

[1] Marietta E, Rishi A, Taneja V. Immunogenetic control of the intestinal microbiota. Immunology. 2015;145(3):313-322. doi:10.1111/ imm.12474.

[2] Neish AS. Mucosal Immunity and the Microbiome. Annals of the American Thoracic Society. 2014;11(Suppl 1):S28-S32. doi:10.1513/AnnalsATS.201306-161MG. 
[3] Sender R, Fuchs S, Milo R. Revised Estimates for the Number of Human and Bacteria Cells in the Body. PLoS Biology. 2016;14(8):e1002533. doi:10.1371/journal. pbio.1002533.

[4] Clemente, J.C., Ursell, L.K., Parfrey, L.W., and Knight, R. (2012). The impact of the gut microbiota on human health: an integrative view. Cell

[5] Sommer, F., and Backhed, F. (2013). The gut microbiota-masters of host development and physiology. Nat. Rev. Microbiol

[6] Hsiao, E.Y., McBride, S.W., Hsien, S., Sharon, G., Hyde, E.R., McCue, T., Codelli, J.A., Chow, J., Reisman, S.E., Petrosino, J.F., et al. (2013). Microbiota modulate behavioral and physiological abnormalities associated with neurodevelopmental disorders. Cell

[7] Shi N, Li N, Duan X, Niu H. Interaction between the gut microbiome and mucosal immune system. Military Medical Research. 2017;4:14. doi:10.1186/s40779-017-0122-9.

[8] Thaiss C.A., Zeevi D., Levy M., Zilberman-Schapira G., Suez J., Tengeler A.C. Transkingdom control of microbiota diurnal oscillations promotes metabolic homeostasis. Cell. 2014;159(3):514-529

[9] de Groot PF, Frissen MN, de Clercq NC, Nieuwdorp M. Fecal microbiota transplantation in metabolic syndrome: history, present and future. Gut Microbes (2017)

[10] Dibner, C., Schibler, U., and Albrecht, U. (2010). The mammalian circadian timing system: organization and coordination of central and peripheral clocks. Annu. Rev. Physiol.

[11] Keller, M., Mazuch, J., Abraham, U., Eom, G.D., Herzog, E.D., Volk, H.D., Kramer, A., and Maier, B. (2009). A circadian clock in macrophages controls inflammatory immune responses. Proc. Natl. Acad. Sci.

[12] Takahashi JS. Transcriptional architecture of the mammalian circadian clock. Nature reviews Genetics. 2017;18(3):164-179. doi:10.1038/nrg.2016.150.

[13] Buxton, O.M., Cain, S.W., O'Connor, S.P., Porter, J.H., Duffy, J.F., Wang, W., Czeisler, C.A., and Shea, S.A. (2012). Adverse metabolic consequences in humans of prolonged sleep restriction combined with circadian disruption. Sci. Transl. Med

[14] Fonken, L.K., Workman, J.L., Walton, J.C., Weil, Z.M., Morris, J.S., Haim, A., and Nelson, R.J. (2010). Light at night increases body mass by shifting the time of food intake. Proc. Natl. Acad. Sc

[15] Savvidis C, Koutsilieris M. Circadian Rhythm Disruption in Cancer Biology. Molecular Medicine. 2012;18(1):1249-1260. doi:10.2119/molmed.2012.00077.

[16] Scheer, F.A., Hilton, M.F., Mantzoros, C.S., and Shea, S.A. (2009). Adverse metabolic and cardiovascular consequences of circadian misalignment. Proc. Natl. Acad. Sci

[17] Swanson, GR., Burgess, HJ. (2017). Sleep and circadian hygiene and inflammatory bowel disease. Gastroenterology Clinics of North America.46(4):881893. doi:10.1016/j.gtc.2017.08.014

[18] Koch S, Nusrat A. The life and death of epithelia during inflammation: lessons learned from the gut. Ann. Rev. Pathol. 2012;7:35-60. doi: 10.1146/annurev-pathol-011811-120905.

[19] Groschwitz KR, Hogan SP. Intestinal Barrier Function: Molecular Regulation and Disease Pathogenesis. The Journal of allergy and clinical immunology. 2009;124(1):3-22. 
[20] Mu Q, Kirby J, Reilly CM, Luo XM. Leaky Gut As a Danger Signal for Autoimmune Diseases. Frontiers in Immunology. 2017;8:598. doi:10.3389/fimmu.2017.00598.

[21] Kiela PR, Ghishan FK. Physiology of Intestinal Absorption and Secretion. Best practice \& research Clinical gastroenterology. 2016;30(2):145-159. doi:10.1016/j. bpg.2016.02.007.

[22] Chakaroun RM, Massier L, Kovacs P. Gut Microbiome, Intestinal Permeability, and Tissue Bacteria in Metabolic Disease: Perpetrators or Bystanders? Nutrients. 2020;12(4). doi:10.3390/nu12041082

[23] Archer, S.N., Laing, E.E., Mo" Iler-Levet, C.S., van der Veen, D.R., Bucca, G., Lazar, A.S., Santhi, N., Slak, A., Kabiljo, R., von Schantz, M., et al. (2014). Mistimed sleep disrupts circadian regulation of the human transcriptome. Proc. Natl. Acad. Sci

[24] Suwazono, Y., Dochi, M., Sakata, K., Okubo, Y., Oishi, M., Tanaka, K., Kobayashi, E., Kido, T., and Nogawa, K. (2008). A longitudinal study on the effect of shift work on weight gain in male Japanese workers. Obesity

[25] Obesity and Overweight. https://www. who.int/news-room/fact-sheets/detail/obesity-and-overweight. Accessed 20 Dec. 2020.

[26] Nojkov, Borko, et al. "The Impact of Rotating Shift Work on the Prevalence of Irritable Bowel Syndrome in Nurses." The American Journal of Gastroenterology, vol. 105, no. 4, Apr. 2010, pp. 842-47. PubMed Central, doi:10.1038/ajg.2010.48.

[27] Thaiss CA, Levy M, Grosheva I, Zheng D, Soffer E, Blacher E, et al. Hyperglycemia drives intestinal barrier dysfunction and risk for 